\title{
Buffalo milk fat globules and their biological membrane: in situ
} structural investigations

Hanh T.H. Nguyen a,b,c, Lydia Ong a,b,c, Eric Beaucher d,e, Marie-Noëlle Madec d,e, Sandra E. Kentish a,c, Sally L. Gras a,b,c, Christelle Lopez d,e,,

a Department of Chemical and Biomolecular Engineering, The University of Melbourne, Parkville, Vic 3010, Australia.

b The Bio21 Molecular Science and Biotechnology Institute, The University of Melbourne, Parkville, Vic 3010, Australia.

${ }^{c}$ The ARC Dairy Innovation Hub, The University of Melbourne, Parkville, Vic 3010, Australia.

${ }^{\mathrm{d}}$ INRA, UMR 1253, Science and Technology of Milk and Egg, F-35000 Rennes, France.

e Agrocampus Ouest, UMR 1253, Science and Technology of Milk and Egg, F-35000 Rennes, France.

* Corresponding author

E-mail address: Christelle.Lopez@ rennes.inra.fr (C. Lopez)

Tel.: +33223485617

Fax: +33223485350

Abbreviations: MFGM: milk fat globule membrane; Lo phase: liquid ordered phase; CLSM: confocal laser scanning microscopy; DIC: differential interference contrast; TAG: triacylglycerol; SM: sphingomyelin; PC: phosphatidylcholine 
Milk fat globules and their surrounding biological membrane (the MFGM) are not well understood despite the importance of these milk components in human nutrition and the role of fat globules in determining the properties of dairy products. The objectives of this study were to investigate these unique colloidal assemblies and the microstructure of the MFGM in buffalo milk, which is the second largest global source of dairy products. In-situ structural investigations were performed at room temperature using confocal microscopy with multiple fluorescent probes (Nile Red, Rh-DOPE, the lectin WGA-488). Microscopic observations showed cytoplasmic crescents around fat globules and the heterogeneous distribution of glycosylated molecules and polar lipids with the occurrence of lipid domains. The lipid domains in the buffalo MFGM appear to form by the segregation of lipids with a high phase transition temperature (e.g. sphingomyelin and saturated phosphatidylcholine molecular temperature lipid, lipid domain 


\section{Introduction}

Milk is an exceptionally complex biological fluid used for the manufacture of a wide range of dairy products. Numerous studies have focused on cow's milk although milks from other animal species such buffalos, ewes, goats and camels are essential to the human diet in various parts of the world. Buffalo milk represents the second largest volume of milk produced globally after cow's milk with more than 97 million tonnes produced each year (FAOSTAT, 2012). Buffalo milk is also one of the richest milks from a compositional point of view (Ménard et al., 2010). Fat constitutes the main fraction of buffalo milk, with the almost twice the fat content of bovine milk (7.4-8.8\% w/w vs. 3.6-4.7\% w/w) (Solh, Staines, Honda, \& Limley, 2007; Varricchio, Di Francia, Masucci, Romano, \& Proto, 2007) and this fat is responsible for the high energetic and nutritive value of buffalo milk. Despite the nutritional value of milk fat and the influence of fat on dairy product properties, information about buffalo milk fat is scarce.

All milk fat is thought to be dispersed in colloidal assemblies called milk fat globules. The core of the milk fat globule is mainly composed of triacylglycerols (TAG, esters of fatty acids and glycerol; $98 \%$ of milk lipids). This core is surrounded by a biological membrane called the milk fat globule membrane (MFGM). This MFGM contains many bioactive compounds which are involved with several biological functions and health benefits, such as neonatal gut maturation, antibacterial infection, inhibition of colon development and lowering cholesterol absorption (Dewettinck et al., 2008; Lopez, 2011). Previous studies have demonstrated that buffalo milk fat globules are significantly larger in size $(5.0 \mu \mathrm{m}$ vs. $3.5 \mu \mathrm{m})$ and have a higher absolute zeta potential $(|-11.0 \mathrm{mV}|$ vs. $|-9.4 \mathrm{mV}|)$ compared to bovine milk fat globules (Ménard et al., 2010). Few authors have characterized the buffalo MFGM (Abou-Dawood, Moussaa, El-Demerdash, \& Ahmed, 1988; D'Ambrosio et al., 2008; Ménard et al., 2010). 
This biological membrane is thought to be comprised of three layers, containing mainly of glycerophospholipids (phosphatidylcholine, PC; phosphatidylethanolamine, PE; phosphatidylinositol, PI; phosphatidylserine, PS), sphingolipids (mainly sphingomyelin, SM), cholesterol and proteins (Keenan \& Patton, 1995; Ménard et al., 2010). Polar lipids, which are mainly located in the MFGM, account for about $2.6 \mathrm{mg} / \mathrm{g}$ fat and about $190 \mathrm{mg} / \mathrm{L}$ of buffalo milk (Ménard et al., 2010), which is $28 \%$ higher than in bovine milk (Ménard et al., 2010). Also, buffalo milk contains less cholesterol than bovine milk (7.0-10.2 mg/100mL vs. 10.519.8 mg/100 mL) (Strzalkowska, Jozwik, Baghnicka, Krzyzewski, \& Horbanczuk, 2009; Talpur, Memom, \& Bhanger, 2007; Zotos \& Bampidis, 2014). The average content of membrane protein was reported to be less in buffalo MFGM compared to bovine milk, either when calculated as a percentage of fat $(4.2 \%$ vs. $4.9 \%)$ or as a proportion of the total MFGM material (29.5\% vs. 33.9\%) (Abou-Dawood et al., 1988). A recent proteomic study identified 50 proteins within buffalo MFGM, with the major proteins being as xanthine dehydrogenase/oxidase, butyrophilin, adipophilin, lactadherin and mucin, similar to bovine MFGM proteins (D'Ambrosio et al., 2008; Fong, Norris, \& MacGibbon, 2007). Both the composition and the structure of the MFGM result from the mechanisms of secretion of fat globules from the epithelial cells of the mammary gland (Heid \& Keenan, 2005). The compositional differences reported to date suggest there may be further underlying structural differences in the MFGM in buffalo milk and possible differences occurring during the in vivo secretion of these globules during milk production.

A number of studies performed using confocal microscopy have revealed heterogeneities in the organization of the MFGM (Evers et al., 2008), in the localization of membrane proteins (Lopez, Madec, \& Jimenez-Flores, 2010; Lopez \& Ménard, 2011) and the presence of lipid domains in the membranes of bovine (Gallier, Gragson, Jimenez-Flores, \& Everett, 2010; Lopez et al., 2011; Lopez et al., 2010) and human milk (Lopez \& Ménard, 2011; Zou et al., 
2012). This heterogeneous distribution has been attributed to the phase separation of polar lipids according to their temperature of phase transition ( $\mathrm{Tm})$. The exogenous phospholipid fluorescently head-labelled with rhodamine (i.e. Rh-DOPE; PE containing 2 oleic acids with very low phase transition temperature) preferentially partitions in the fluid Ld phase containing unsaturated polar lipids (i.e. containing one or two long-chain unsaturated fatty acids) (Lopez et al., 2010). Hence, areas of the MFGM not stained by Rh-DOPE may correspond to gel phase domains rich in polar lipids with a high Tm. Polar lipids not stained by Rh-DOPE could adopt a second possible phase consisting of liquid ordered (Lo) phase domains composed of high Tm lipids and cholesterol. These high Tm lipids include SM, which accounts for about 25\% of polar lipids in the MFGM (Lopez, 2011) and contains long chain saturated fatty acids ( $\mathrm{Tm}=35^{\circ} \mathrm{C}$; Malmsten, Bergentahl, Nyberg, and Odham (1994)). Saturated PC are also high Tm lipids found in the MFGM (e.g. dipalmitoyl phosphatidylcholine, DPPC, $\mathrm{Tm}=41.7{ }^{\circ} \mathrm{C}$; Benesch and McElhaney (2014)). The surrounding membrane stained by Rh-DOPE may correspond to the fluid liquid disordered (Ld) phase of the unsaturated glycerophospholipids (PC, PE, PI, PS) which have a low Tm and are responsible for the fluidity of the biomembranes (Gallier et al., 2010; Lopez et al., 2010). A recent study performed by atomic force microscopy with a model membrane showed that milk SM segregates from the fluid Ld phase of unsaturated polar lipids to form domains in the gel phase (Guyomarc'h et al., 2014). This study also showed that cholesterol has a large effect on the domains formed by milk SM; the shapes and nanomechanical properties of the domains formed differ in the gel phase in the absence of cholesterol and in the Lo phase when cholesterol is present. The domains also protrude further from the fluid phase of the membrane in the absence of cholesterol (Guyomarc'h et al., 2014).

The lipid domains recently revealed in the MFGM could potentially be responsible for several biological processes (e.g. digestion of milk fat globules, interaction with pathogens and 
viruses in the gut) and cellular functions. Also, the lipid domains present in outer bilayer of the MFGM could be involved in functional properties of milk fat globules, important for dairy applications.

The objective of this study was to perform a structural analysis of fat globules and their biological membrane, in situ in buffalo milk. The microstructure was investigated using the combination of optical microscopy with differential interferential contrast together with confocal laser scanning microscopy (CLSM) with adapted fluorescent dyes able to label TAG, total proteins, polar lipids and glycosylated molecules located in the MFGM.

\section{Materials and methods}

\subsection{Milk samples}

The buffalo milks used in this study were a mixture of the individual milks produced by 30 buffaloes of the Mediterranean breed Bubalus bubalis and collected from evening and morning milking from Coopérative de Bufflonnes (Maurs, Cantal region, France). $\mathrm{NaN}_{3}$ $(0.02 \% \mathrm{w} / \mathrm{v})$ was added to the milk to prevent the growth of bacteria. Milk samples were stored at ambient temperature before fat globule size measurements and CLSM experiments. The content of fat in the milk was determined using the Gerber method method (IDF, 2008).

\subsection{Fat globule size measurements}

2.3 The fat globule size distributions were determined by laser light scattering, using a Mastersizer 2000 (Malvern Instruments, Malvern, U.K.) equipped with a $\mathrm{He} / \mathrm{Ne}$ laser $(\lambda=$ $633 \mathrm{~nm})$ and an electroluminescent diode $(\lambda=466 \mathrm{~nm})$. The refractive indexes of milk fat 
were set at 1.46 (at $466 \mathrm{~nm}$ ) and 1.458 (at $633 \mathrm{~nm}$ ) and the refractive index was set at 1.33 for water. The absorption coefficient used was 0.0001 . The experiments were performed at room temperature. Aliquots of approximately $70 \mu \mathrm{L}$ of whole milk were introduced into the measurement cell of the apparatus, which contained $100 \mathrm{ml}$ of water, in order to reach $10 \%$ obscuration (optimal conditions for particle-size measurements with this apparatus). A solution of $35 \mathrm{mM}$ EDTA/NaOH pH 7.0 buffer (>98\% disodium salt dihydrat, Prolabo, Fortenay-sous-Bois, France) was added to the measurement cell to disrupt the casein analysis

Microstructural analysis was performed using an inverted microscope NIKON EclipseTE2000-C1si (NIKON, Champigny sur Marne, France). Confocal laser scanning microscopy (CLSM) was performed using an argon laser operating at an excitation wavelength of $488 \mathrm{~nm}$ with emission detected between $500 \mathrm{~nm}$ and $530 \mathrm{~nm}$, a He-Ne laser operating at 543nm wavelength excitation with emission detected between $565 \mathrm{~nm}$ and $615 \mathrm{~nm}$ and a diode operating at $633 \mathrm{~nm}$, detected with a long pass filter $>650 \mathrm{~nm}$. The observations were performed using a $\times 100$ (numerical aperture NA 1.4) oil immersion objective.

The staining protocols followed previously described methods (Lopez et al., 2010; Lopez \& Ménard, 2011). Briefly, Nile Red (5H-Benzo $\alpha$-phenoxazine-5-one, 9-diethylamino, supplied by Sigma-Aldrich, St. Louis, USA) was prepared at a concentration of $42 \mu \mathrm{g} / \mathrm{mL}$ in propanediol and used to stain the triacylglycerol core of the fat globules. Fast Green FCF 
used to stain proteins. N-(Lissamine rhodamine B sulfonyl) dioleoylphosphatidyl ethanolamine (Rh-DOPE, Avanti polar lipids Inc., Birmingham, England) was provided at a concentration of $1 \mathrm{mg} / \mathrm{mL}$ in chloroform and used to label the phospholipids in the membrane surrounding buffalo milk fat globules. Wheat germ agglutinin Alexa fluor 488 (WGA488, Invitrogen, Cergy Pontoise, France) was prepared at a concentration of $1 \mathrm{mg} / \mathrm{mL}$ in phosphate saline buffer and used to label the glycosylated molecules in the membrane, i.e. carbohydrate moieties containing $\mathrm{N}$-acetylglucosamine and $\mathrm{N}$-acetyl neuraminic acid (sialic acid) residues.

The concentrations of the fluorescent dyes were adapted to the specific composition of buffalo milk. For observation of the fat globules, $100 \mu \mathrm{L}$ of Nile Red and $10 \mu \mathrm{L}$ of Fast Green FCF were added to $1 \mathrm{~mL}$ of buffalo milk samples. For observation of the MFGM polar lipids, $40 \mu \mathrm{L}$ of the Rh-DOPE solution was placed in a glass vial and the chloroform was evaporated under nitrogen, to avoid the possible artefacts caused by this organic solvent. Then $1 \mathrm{~mL}$ of milk sample was introduced in the vial. For dual staining of the MFGM polar lipids and carbohydrate moieties, $10 \mu \mathrm{L}$ of the WGA-488 solution was also added into the vial containing Rh-DOPE. Then, $1 \mathrm{~mL}$ of milk sample was added to the vial. The stained milk samples were kept at room temperature for at least $1 \mathrm{~h}$ prior to observation by CLSM. The microstructural analyses were performed at room temperature $\left(19 \pm 1{ }^{\circ} \mathrm{C}\right)$

Optical microscopy using differential interference contrast (DIC, also called Nomarski, Cogswell and Sheppard (1992)) was also used to characterize buffalo milk fat globules. DIC images were superimposed on the fluorescent emission recorded in the CLSM images. The two dimensional images had a resolution of $512 \times 512$ pixels and the pixel scale values were converted into micrometers using a scaling factor. In the multiple labeled samples, different colors were used to locate the fluorescent probes (e.g. red color for phospholipids stained by 
Rh-DOPE, green color for the carbohydrate moieties stained by WGA-488 and grey levels for DIC images).

\section{Results and discussion}

\subsection{Size distribution of buffalo milk fat globules}

The buffalo milks used in this study contained $74.1 \pm 5.8 \mathrm{~g}$ fat per $\mathrm{kg}$ milk, in agreement with previous studies (Ahmad et al., 2008; Ménard et al., 2010; Varricchio et al., 2007). The size distribution of the fat globules, determined using light scattering, ranged from $0.4 \mu \mathrm{m}$ to 15.8 $\mu \mathrm{m}$, with a mean volume-weighted diameter of $5.0 \pm 0.4 \mu \mathrm{m}$ (Figure 1A). The surface of fat globules covered by the MFGM was $2.0 \pm 0.2 \mathrm{~m}^{2} / \mathrm{g}$ fat, which corresponded to about $148 \mathrm{~m}^{2}$ per kg milk. The biological membrane surrounding fat globules represents a large interface between the TAG core of fat globules and the aqueous environment, confirming the importance of its characterization. These size parameters are consistent with previous reports (Ahmad et al., 2008; Ménard et al., 2010).

The fat globules, observed by the combination of differential interference contrast (DIC) light microscopy and CLSM after staining with Nile Red, were spherical in shape and dispersed in the aqueous phase of milk containing proteins (Figure 1B and D). A rough surface could be observed on some fat globules (indicated by arrows in Figure 1C) and may arise from surface heterogeneities or partial crystallisation of the TAG core. The overlay of DIC and fluorescent CLSM images showed that the triacylglycerols are located in the core of fat globules (Figure 1D), similar to previous observations for bovine and human milk (Lopez et al., 2010; Lopez \& Ménard, 2011; Zou et al., 2012). 
210

211

212

213

214

215

216

217

218

219

220

Cytoplasmic remnants (CR) from lactating cells were observed to be connected to some buffalo fat globules of various sizes, using optical microscopy and fluorescence microscopy (Figure 2). The volume of CR varied from one globule to another and their thickness was 1 $\mu \mathrm{m}$ up to $5 \mu \mathrm{m}$, as determined in the fluorescence images (Figure 2). The CR can exceed the buffalo milk fat globule volume (Figure $2 \mathrm{D}$ ). The morphology of CR can be compared to a crescent attached to fat globules, consistent with another name for these remnants of cytoplasmic crescents. The majority of the buffalo fat globules did not contain CR and were enveloped compactly by the MFGM (Figure $2 \mathrm{D}$ and E).

CR occur when the mechanisms involved in the closure of the apical plasma membrane behind the projecting lipid droplet lead to their secretion with a piece of cytoplasm remaining attached to fat globule in milk (Heid \& Keenan, 2005). The occurrence of the CR has also been reported in previous CLSM studies of human milk (Evers et al., 2008; Lopez \& Ménard, 2011) but CR have not been observed in bovine milk (Lopez et al., 2010) when the same CLSM methods and fluorescent probes have been applied. This is probably due to variations in $\mathrm{CR}$ concentration between the milk of different species, arising from differences in the membrane secretion process, which is poorly understood (Robenek et al., 2005). CR were observed for $7.2 \%$ of the fat globules within human milk but for less than $1 \%$ of the fat globules in bovine milk, quantified using microscopic observation with the assistance of a counting chamber slide (Huston \& Patton, 1990). In goat milk, between 1\% and 5\% of milk fat globules had CR (Wooding, Peaker, \& Linzell, 1970). The current study is the first to report the presence of $\mathrm{CR}$ in buffalo milk but the number of $\mathrm{CR}$ was not quantified, which may be worthy of further investigation. 
Previous authors who observed CR in human milk employed Rh-DOPE or $\mathrm{DiIC}_{18}$ (3)-DS to label the MFGM (Evers et al., 2008; Lopez \& Ménard, 2011). DiIC18(3)-DS is a lipophilic fluorescent probe that can penetrate the membrane by inserting its two acyl chains into the interior of the phospholipid membrane (Evers et al., 2008). The labelling of the CR by RhDOPE or DiIC18(3)-DS suggests that these remnants are rich in phospholipid, consistent with their origin as fragments of the cytoplasm or apical plasma membrane (Heid \& Keenan, 2005). The size of CR has been observed to range from a thin sliver to a size exceeding the fat globule core (Heid \& Keenan, 2005). CR contain all organelles and intracellular membrane materials (except for nuclei) of the lactating cells (Heid \& Keenan, 2005; Wooding et al., 1970) and thus, these CR may be a good source of enzymes, micronutrients and growth factors necessary for newborn development (Huston \& Patton, 1990). RNA has also been successfully isolated from the CR of human milk (Lemay et al., 2013; Maningat et al., 2009), suggesting CR could impact on protein expression in host cells, similar to other RNA containing membrane vesicles found in milk and other body fluids (Valadi et al., 2007; Zhou et al., 2012). CR could similarly be examined as potential markers of milk quality.

\subsection{Heterogeneous distribution of polar lipids in buffalo MFGM}

The fluorescent dye Rh-DOPE was used to assess the distribution of polar lipids in the MFGM surrounding buffalo fat globules, in situ in buffalo milk at $19 \pm 1{ }^{\circ} \mathrm{C}$, as already performed for bovine and human MFGM (Gallier et al., 2010; Lopez et al., 2010; Lopez \&

Ménard, 2011; Zou et al., 2012).

Rh-DOPE integrated the buffalo MFGM and appeared fluorescent at the periphery of the fat

253 globules viewed as an equatorial section by CLSM (Figure 3, Figure 4 A and C). The interior 254 of buffalo milk fat globules, which is mainly composed of triacylglycerols, as shown in 255 Figure 1, was not labelled by Rh-DOPE (Figure 3 and Figure 4 C). In some cases, the entire 
periphery of fat globules was fluorescent, showing the homogeneous distribution of the fluorescent dye, while in other cases, both fluorescent and non-fluorescent areas were visible, indicating the partial labelling of the MFGM by the exogenous phospholipid Rh-DOPE. Three dimensional observations (i.e. recording of thin optical sections at different z-depths of milk samples) allowed the characterisation of non-fluorescently-labelled domains at the surface of milk fat globules composed by the MFGM (noted Z-depth 1 in Figure 4A) and of fluorescent rings seen in an equatorial cross section of the fat globule (noted Z-depth 2 in Figure 4B). These non-fluorescent areas were mostly circular in shape with a size of approximately 0.5 to $2 \mu \mathrm{m}$ and a number generally ranging from zero to nine in one side of fat globule (Figures 4). Differences in size and shape of the non-fluorescent areas, however, were also observed (Figure 4). For example, Figure 4 shows fat globules with circular non-fluorescent areas of size ranging from very small (Figure 4 B) to very large (Figure 4 E), while fat globules with irregular shaped non-fluorescent regions have also been characterized (Figure 4 F, G, H). The irregular shapes could result from the connection of several circular domains at the surface of fat globules or to different physical states of the lipids in the domains. Non-fluorescent domains not stained by the Rh-DOPE can diffuse in the plane of the membrane as a function of time, as observed at the surface of a fat globule (highlighted by arrows in Figure 5). This result shows that the MFGM is a highly dynamic system, as previously reported for the bovine MFGM (Lopez et al., 2010).

\subsection{Simultaneous localisation of polar lipids, glycoproteins and glycolipids in buffalo} MFGM

The organisation of lipids and the localisation of the MFGM glycoproteins and glycolipids, in situ around buffalo milk fat globules, was investigated by CLSM with the simultaneous labelling of phospholipids with Rh-DOPE that stain the Ld phase and glycosylated molecules 
that are specifically probed using the fluorescently labelled lectin wheat germ agglutinin (WGA-488). DIC optical microscopy in combination with CLSM permitted the observation of fat globules and the localisation of the components in the MFGM surrounding fat globules.

Figure 6 shows the heterogeneous lateral organisation of lipids and glycoproteins in the MFGM. Figure 6 A focuses on the top view of a fat globule (noted Z-depth 1) of about $10 \mu \mathrm{m}$ diameter, while Figure 6 B-D shows an equatorial cross section of the same fat globule (at a higher Z-depth, noted Z-depth 2), with TAG in the core and the MFGM at the periphery. The lectin stain WGA-488 is reported to bind to N-acetylglucosamine and N-acetylneuraminic acid residues (Evers et al., 2008; Lopez et al., 2010), including the glycolipids such as gangliosides and the main glycoproteins of the MFGM such as butyrophilin, mucin and lactadherin (Evers et al., 2008; Heid \& Keenan, 2005; Lopez et al., 2010). The distribution of these glycosylated molecules is non-homogeneous occurring in patches or networks in some areas of the MFGM of buffalo milk fat globules (Figure 6 and 7).

The co-localisation of polar lipids and glycosylated molecules reveals that most of the nonfluorescent areas observed when the MFGM was labelled with Rh-DOPE were also not fluorescent after labelling with WGA-488 (Figure 6). This is an additional argument in favour of the interpretation of the domains in which Rh-DOPE did not integrate, as being domains rich in SM and DPPC (i.e. high Tm lipids that are not glycosylated). Some exceptions were observed, however, where areas that were not stained by Rh-DOPE but stained by WGA-488 (areas indicated by arrows in Figure 7), indicating that some glycoproteins and/or glycolipids are located in the high Tm lipid rich domains. Also, some glycosylated molecules were observed at the periphery of the domains not labelled by Rh-DOPE (Figure 8). These results suggest that the glycosylated molecules are mostly present in the fluid phase of the MFGM but they can also participate in the high Tm lipid domains at times. Figure 7 E shows that 
glycosylated molecules protrude in the aqueous phase surrounding buffalo milk fat globules, and contribute in the formation of a glycocalyx.

The heterogeneous distribution of glycosylated molecules has also been reported in previous studies for bovine, sheep and human milk (Evers et al., 2008; Gallier et al., 2010; Lopez et al., 2011; Lopez et al., 2010). Non-fluorescent regions have been observed within human and bovine MFGM when dual-staining with Rh-DOPE and WGA-488 has been applied (Gallier et al., 2010; Lopez et al., 2011; Lopez et al., 2010; Lopez \& Ménard, 2011) and these non-fluorescent areas were attributed to SM-rich domains. Non-fluorescent areas were also observed within the bovine, sheep and human MFGM when the milk was single stained with WGA488 or when dual stained with WGA488 and the lipophilic probe FMN-64 (Evers et al., 2008), although these studies interpreted these non-fluorescent areas as the regions where the MFGM is absent. The lateral organisation of polar lipids and proteins in the MFGM could be involved in specific functions (including the mechanisms of lipid digestion or protection of the neonates from infections), which currently remain unknown and require further investigation.

\subsection{The MFGM: a non-random mixing of components}

We observed that the MFGM is a non-random mixture of components characterised by the lateral separation of compositionally distinct lipid phases. The occurrence of both fluorescent and non-fluorescent areas observed with Rh-DOPE staining was interpreted as a heterogeneous distribution of the polar lipids within the buffalo MFGM. Phase separation would result in preferential partition of select lipids in the coexisting phases as a function of their chemical and physical properties. Such heterogeneities in the partitioning of Rh-DOPE stained lipids have been previously observed in the human and bovine MFGM (Lopez et al., 2011; Lopez et al., 2010; Lopez \& Ménard, 2011; Zou et al., 2012). 
The non-fluorescent areas characterised at room temperature for the buffalo MFGM could correspond to the preferential tight packing of polar lipids with a high gel to liquid crystalline phase transition temperature (high $\mathrm{Tm}$ ), leading to their lateral segregation from very low Tm polar lipids in the liquid phase of the MFGM. These high Tm polar lipids consist of longchain saturated fatty acids (i.e. C16:0, P; C18:0, S; and fatty acids with a number of carbon > 20). According to the fatty acid composition of polar lipids in the MFGM (Sanchez-Juanes, Alonso, Jancada, \& Hueso, 2009) and the preferential concentration of PC and SM in the outer layer of the MFGM (Deeth, 1997), these polar lipids could be SM (the main fatty acids being C16:0, C20:0, C22:0, C23:0, C24:0; Lopez (2011)) and PC (i.e. DPPC, PSPC or SSPC). Under the conditions examined $\left(\mathrm{T}=19^{\circ} \mathrm{C}\right)$, these milk SM and saturated PC molecular species are expected to be in the gel phase, as they have a $\mathrm{Tm}>19{ }^{\circ} \mathrm{C}$. Such high Tm polar lipids can also segregate together with cholesterol in the plane of the biological membranes to form ordered lipid domains in the Lo phase (Brown \& London, 1998). The role played by cholesterol in the MFGM is important, since a minimum molar percentage of cholesterol is necessary to form the Lo phase with high Tm lipids, as reported in phase diagrams built with controlled lipid compositions (Veatch \& Keller, 2005). The coexistence of two ordered phases (i.e. the Lo phase domains including high Tm lipids and cholesterol and the gel phase domains composed only by high Tm lipids) with one fluid phase composed by unsaturated polar lipids may occur, as already discussed for ternary model systems (Veatch \& Keller, 2005). The ordered phases (gel and Lo phases) are immiscible with the MFGM fluid phase and form micron-scale domains, permitting their observation by confocal microscopy. The circular shape of the domains observed in the buffalo MFGM at room temperature is consistent with the formation of Lo phase domains, since polar lipids organised in a gel phase are semi-crystallised and form angles (Brown \& London, 1998; Morales-Penningston et al., 2011). Non-circular domains present in the outer bilayer of MFGM have been previously 
reported at room temperature for human milk (Lopez \& Ménard, 2011) but have not been observed for the bovine MFGM (Lopez et al., 2010). This difference in the morphology of the lipid domains observed in the MFGM from various species could possibly be due to the differences in the composition of the polar lipids such as the concentration and individual lipid species with high Tm (for example SM and DPPC) and the relative proportion of low Tm lipids, high Tm lipids and cholesterol (Veatch \& Keller, 2005). The relative proportion of $\mathrm{SM}$, which is assumed to be the major component of the Lo phase domains (Gallier et al., 2010; Lopez et al., 2010), is lower in the buffalo MFGM compared to the bovine MFGM ( $24.8 \%$ vs. $26.9 \%$ of polar lipid) (Ménard et al., 2010). It has been reported that the morphology of the SM-rich domains and the composition of the bovine MFGM could be linked to fat globule size (Gallier et al., 2010; Lopez et al., 2011). Although some studies have reported that small bovine fat globules appear to have fewer domains compared to large fat globules (Gallier et al., 2010), others did not find any significant differences arising from the characterisation of domains in small fat globules (diameter $<2 \mu \mathrm{m}$ ) (Lopez et al., 2011). The lipid composition of the outer bilayer of the MFGM could be considered as a ternary system consisting of high Tm polar lipids (e.g. SM and saturated PC), cholesterol and low Tm polar lipids (e.g. unsaturated glycerophospholipids) with differences in membrane structure and dynamics arising from compositional differences between species.

The organisation of lipids in the MFGM can govern the functional properties of this biomembrane, affect the localisation of membrane-associated proteins and the chemical reactions that occur in the MFGM or that are mediated by this membrane. Lipid phase separation could also influence the binding of molecules to the MFGM, such as bacteria and viruses (Lopez, 2011). The MFGM plays a key role in the physical stability of fat globules in milk including protection against coalescence and aggregation. Buffalo milk fat globules are 
important in the preparation of dairy products such as yoghurt and cheese. Hence, the lipid domains formed in the MFGM, could be involved in a number of functions, warranting further investigation of these microstructural features.

\section{Conclusion}

Buffalo fat globules and their biological membrane are poorly characterised despite the importance of buffalo milk to human nutrition and dairy processing. This study investigated the microstructure of the buffalo MFGM improving our knowledge of this dynamic and complex biological membrane. We showed that the MFGM is heterogeneous with respect to lipid, cholesterol and protein assemblies. Confocal experiments revealed that polar lipids segregated in the outer bilayer of the MFGM into two or more liquid phases (e.g. gel, Lo and Ld phases) that can coexist and have different physical properties, with gel and Ld phases being most probable. Glycoproteins and glycolipids were mainly present in the Ld fluid phase but also existed in the lipid domains (gel or Lo phases) at times. Whether or not the lipid domains in buffalo MFGM are lipid rafts remains unknown and would be worthy of further study.

\section{Acknowledgements}

Joëlle Léonil, the head of INRA STLO (Rennes, France), is acknowledged for hosting Hanh Nguyen (PhD student). The authors thank Joël Guillemin (Coopérative de Bufflonnes, Maurs, France) for providing buffalo milks. The authors also acknowledge The Australian Government including The ARC Dairy Innovation Hub, The Rural Industries Research and Development Cooperation (RIRDC), The University of Melbourne, The Bio21 Molecular 
Science and Biotechnology Institute, The Particulate Fluids Processing Centre (PFPC) and The Clive Pratt Family for financial support.

All authors have approved the final article.

\section{References}

Abou-Dawood, A. E., Moussaa, A. E., El-Demerdash, O., \& Ahmed, R. B. (1988). Comparative study on fat globule membrane of buffalo, cow, goat and sheep raw milk. Annals of Agricultural Science, 26(4), 2527-2536.

Ahmad, S., Gaucher, I., Rousseau, F., Beaucher, E., Piot, M., Grongnet, J. F., \& Gaucheron, F. (2008). Effects of acidification on physico-chemical characteristics of buffalo milk: A comparison with cow's milk. Food Chemistry, 106(1), 11-17.

Benesch, M. G. K., \& McElhaney, R. N. (2014). A comparative calorimetric study of the effects of cholesterol and the plant sterols campesterol and brassicasterol on the thermotropic phase behavior of dipalmitoylphosphatidylcholine bilayer membranes. Biochimica et Biophysica Acta (BBA) - Biomembranes, 1838(7), 1941-1949.

Brown, D. A., \& London, E. (1998). Functions of lipid rafts in biological membranes. Annual Review of Cell and Developmental Biology, 14, 111-136.

Cogswell, C. J., \& Sheppard, C. J. R. (1992). Confocal differential interference contrast (DIC) microscopy: Including a theoretical analysis of conventional and confocal DIC imaging. Journal of Microscopy, 165(1), 81-101.

D'Ambrosio, C., Arena, S., Salzano, A. M., Renzone, G., Ledda, L., \& Scaloni, A. (2008). A proteomic characterization of water buffalo milk fractions describing PTM of major species and the identification of minor components involved in nutrient delivery and defense against pathogens. Proteomics, 8, 3657-3666.

Deeth, H. C. (1997). The role of phospholipids in the stability of milk fat globules. Australian Journal of Dairy Technology, 52(1), 44-46.

Dewettinck, K., Rombaut, R., Thienpont, N., Le, T. T., Messens, K., \& Camp, J. V. (2008). Nutritional and technological aspects of milk fat globule membrane material. International Dairy Journal, 18, 436-457.

Evers, J. M., Haverkamp, R. G., Holroyd, S. E., Jameson, G. B., Mackenzie, D. D. S., \& McCarthy, O. J. (2008). Heterogeneity of milk fat globule membrane structure and composition as observed using fluorescence microscopy techniques. International Dairy Journal, 18(12), 1081-1089. 
FAOSTAT. (2012). Production quantity of livestock primary 2012. Food and Agriculture Organisation of the United Nations - Statistics Division. Retrieved 23/10/2014 from http://faostat3.fao.org/download/Q/QL/E.

Fong, B. Y., Norris, C. S., \& MacGibbon, A. K. H. (2007). Protein and lipid composition of bovine milk-fat-globule membrane. International Dairy Journal, 17, 275-288.

Gallier, S., Gragson, D., Jimenez-Flores, R., \& Everett, D. (2010). Using confocal laser scanning microscopy to probe the milk fat globule membrane and associated proteins. Journal of Agricultural and Food Chemistry, 58(7), 4250-4257.

Guyomarc'h, F., Zou, S., Chen, M., Milhiet, P. E., Godefroy, C., Vié, V., \& Lopez, C. (2014). Milk sphingomyelin domains in biomimetic membranes and the role of cholesterol: morphology and nanomechanical properties investigated using AFM and force spectroscopy. Langmuir, 30(22), 6516-6524.

Heid, H. W., \& Keenan, T. W. (2005). Intracellular origin and secretion of milk fat globules. European Journal of Cell Biology, 84(2-3), 245-258.

Huston, G. E., \& Patton, S. (1990). Factors related to the formation of cytoplasmic crescents on milk fat globules. Journal of Dairy Science, 73(8), 2061-2066.

IDF. (2008). Milk - Determination of fat content (Gerber method). (International standard ISO-IDF 2446:2008). Brussels, Belgium: Interntationdal Dairy Federation.

Keenan, T. W., \& Patton, S. (1995). The structure of milk: implications for sample and storage. A. The milk lipid globule membrane. In R. G. Jensen (Ed.), Handbook of milk composition (pp. 5-49). San Diego, CA, USA: Academic Press.

Lemay, D. G., Ballard, O. A., Hughes, M. A., Morrow, A. L., Horseman, N. D., \& NommsenRivers, L. A. (2013). RNA sequencing of the human milk fat layer transcriptom reveals distinct gene expression profiles at three stages of lactation. Plosone, 8(7). doi: 10.1371/journal.pone.0067531. Retrieved 25/09/2014 from http://www.plosone.org/article/info\%3Adoi\%2F10.1371\%2Fjournal.pone.0067531.

Lopez, C. (2011). Milk fat globules enveloped by their biological membrane: Unique colloidal assemblies with a specific composition and structure. Current Opinion in Colloid \& Interface Science, 16(5), 391-404.

Lopez, C., Briard-Bion, V., Menard, O., Beaucher, E., Rousseau, F., Fauquant, J., . . Robert, B. (2011). Fat globules selected from whole milk according to their size: Different compositions and structure of the biomembrane, revealing sphingomyelin-rich domains. Food Chemistry, 125(2), 355-368.

Lopez, C., Madec, M. N., \& Jimenez-Flores, R. (2010). Lipid rafts in the bovine milk fat globule membrane revealed by the lateral segregation of phospholipids and heterogeneous distribution of glycoproteins. Food Chemistry, 120(1), 22-33.

Lopez, C., \& Ménard, O. (2011). Human milk fat globules: Polar lipid composition and in situ structural investigations revealing the heterogeneous distribution of proteins and the lateral segregation of sphingomyelin in the biological membrane. Colloids and Surfaces B: Biointerfaces, 83(1), 29-41. 
Malmsten, M., Bergentahl, B., Nyberg, L., \& Odham, G. (1994). Sphingomyelin from milk characterisation of liquid crystalline, liposome and emulsion properties. Journal of the American Oil Chemists Society, 91(9), 1021-1026.

Maningat, P. D., Sen, P., Rijnkels, M., Sunehag, A. L., Hadsell, D. L., Bray, M., \& Haymond, M. W. (2009). Gene expression in the human mammary epithelium during lactation: the milk fat globule transcriptome. Physiological Genomics, 37(1), 12-22.

Ménard, O., Ahmad, S., Rousseau, F., Briard-Bion, V., Gaucheron, F., \& Lopez, C. (2010). Buffalo vs. cow milk fat globules: Size distribution, zeta-potential, compositions in total fatty acids and in polar lipids from the milk fat globule membrane. Food Chemistry, 120(2), 544-551.

Morales-Penningston, N. F., Wu, J., Farkas, E. R., Goh, S. L., Konyakhina, T. M., Zheng, J. Y., . . Feigenson, G. W. (2011). GUV preparation and imaging: minimizing artifacts. Biochimica et Biophysica Acta, 1798(7), 1324-1332.

Robenek, H., Robenek, J. M., Buers, I., Lorkowski, S., Hofnagel, O., Troyer, D., \& Severs, N. J. (2005). Lipid droplets gain PAT family proteins by interaction with specialised plasma membrane domains. The Journal of Biological Chemistry, 280, 26330-26338.

Sanchez-Juanes, F., Alonso, J. M., Jancada, L., \& Hueso, P. (2009). Distribution and fatty acid content of phospholipids from bovine milk and bovine milk fat globule membranes. International Dairy Journal, 19, 273-278.

Solh, V. A., Staines, V., Honda, S., \& Limley, H. A. (2007). Measurement of milk color and composition: Effect of dietary intervention on western Australian Holstein-Friesian cow's milk quality. Journal of Food Science, 72(8), 560-566.

Strzalkowska, N., Jozwik, A., Baghnicka, E., Krzyzewski, J., \& Horbanczuk, J. O. (2009). Studies upon genetic and environmental factors affecting the cholesterol content of cow milk. I. Relationship between the polymorphic form of beta-lactoglobulin, somatic cell count, cow age and stage of lactation and cholesterol content of milk. Animal Science Papers and Reports, 27(2), 95-103.

Talpur, F. N., Memom, N. N., \& Bhanger, M. I. (2007). Comparison of fatty acid and cholesterol content of Pakistani water buffalo breeds. Pakistan Journal of Analytical and Environmental Chemistry, 8, 15-20.

Valadi, H., Ekstrom, K., Bossios, A., Sjostrand, M., Lee, J. J., \& Lotvall, J. O. (2007). Exosome-mediated transfer of mRNAs and microRNAs is a novel mechanism of genetic exchange between cells. Nature Cell Biology, 9(6), 654-659.

Varricchio, M. L., Di Francia, A., Masucci, F., Romano, R., \& Proto, V. (2007). Fatty acid composition of Mediterranean buffalo milk fat. Italian Journal of Animal Science, 6, 509-511.

Veatch, S. L., \& Keller, S. L. (2005). Seeing spots: Complex phase behavior in simple membranes. Biochimica et Biophysica Acta (BBA) - Molecular Cell Research, 1746(3), 172-185. 
Wooding, F. B. P., Peaker, M., \& Linzell, J. L. (1970). Theories of milk secretion : evidence from the electron microscopic examination of milk. Nature, 226(5247), 762-764.

Zhou, Q., Li, M., Wang, X., Li, Q., Wang, T., Zhu, Q., . . Li, X. (2012). Immune-related microRNAs are abundant in breast milk exosomes. International Journal of Biological Sciences, 8(1), 118-123.

Zotos, A., \& Bampidis, V. A. (2014). Milk fat quality of Greek buffalo (Bubalus bubalis). Journal of Food Composition and Analysis, 33(2), 181-186.

Zou, X. Q., Guo, Z., Huang, J. H., Jin, Q. Z., Cheong, L. Z., Wang, X. G., \& Xu, X. B. (2012). Human milk fat globules from different stages of lactation: a lipid composition analysis and microstructure characterisation. Journal of Agricultural and Food Chemistry, 60, 7158-7167.

\section{FIGURE CAPTION}

Figure 1: Size distribution of buffalo milk fat globules determined using light scattering (A) in combination with microscopy (B-D). (B) CLSM image with Nile Red labeled fat globule cores appearing red and Fast Green FCF labeled protein appearing green, (C) Image taken with differential interferential contrast and (D) overlay of images A and B. The scale bars are $10 \mu \mathrm{m}$ in length. Arrows indicate fat globules with a rough surface.

Figure 2: Microscopy images showing the presence of cytoplasmic remnants (CR) attached to buffalo milk fat globules. (A) DIC image, (B) CLSM image of fat globules stained with Rh-DOPE, (C) overlay of images A and B, (D and E) CLSM images with Rh-DOPE. Images were captured using a 100x objective. The scale bars are indicated in the images. Thick arrows indicate cytoplasmic remnant, thin arrows show the non-fluorescent domain in the biological membrane surrounding fat globules. TAG core $=$ triacylglycerol core of milk fat globules.

Figure 3: Microscopy images showing an equatorial cross-section of fat globules and the heterogeneous distribution of polar lipids within the buffalo MFGM surrounding the milk fat 
globules. (A) CLSM image after labelling with Rh-DOPE (red colour), (B) overlay of DIC

548 optical microscopy image and CLSM image. The non-fluorescent areas (dark areas) observed around the fat globules correspond to the lateral segregation of high phase transition temperature polar lipids in domains (indicated by the white arrows). Images were captured at

551

552

553

554

555

556

557

558

559

560

561

562

563

564

565

566

567

568

569

570

571

572

573

574

575

576

577

578

579

580 room temperature $\left(19 \pm 1{ }^{\circ} \mathrm{C}\right)$ using a $100 x$ objective. The scale bars are indicated in the figures.

Figure 4: Microscopy images showing the heterogeneous distribution of polar lipids within the buffalo MFGM. (A, B, C) CLSM images after labelling with Rh-DOPE (red colour), (DH) overlay of DIC optical microscopy image and CLSM image. The non-fluorescent areas (dark areas) observed around the fat globules correspond to the lateral segregation of high phase transition temperature polar lipids in domains (indicated by the white arrows). Images were captured at room temperature $\left(19 \pm 1^{\circ} \mathrm{C}\right)$ using a $100 \mathrm{x}$ objective. The scale bars are indicated in the figures.

Figure 5: CLSM images taken at room temperature showing the diffusion of a nonfluorescent lipid domain within the MFGM as a function of time (A) $t=0 \mathrm{sec},(B) t=30 \mathrm{sec}$. The polar lipids within the MFGM were stained using Rh-DOPE (red colour). The scale bars are indicated in the figures.

Figure 6: Microscopy images showing the heterogeneous distribution of polar lipids and glycosylated molecules (glycoproteins and glycolipids) in the buffalo milk fat globule membrane characterised at different z-depths. In-situ observations performed by the combination of DIC optical microscopy and CLSM, using Rh-DOPE to label the phospholipids (red colour) and the lectin WGA-488 to stain the glycosylated molecules (green colour). (A) overlay of DIC and fluorescence (Rh-DOPE and WGA) at z-depth 1 corresponding to the polar region of fat globules, $(\mathbf{B})$ overlay of DIC and fluorescence $(\mathrm{Rh}-$ DOPE and WGA-488) at z-depth 2 corresponding to the equatorial section of fat globules, $(\mathbf{C})$ overlay of DIC and CLSM image with WGA-488, (D) overlay of DIC and CLSM image with Rh-DOPE. Images were captured using a 100x objective. The scale bars are indicated in the figures. White arrows correspond to non-fluorescent domains, green arrows correspond to glycosylated molecules located at the periphery or within a domain in which Rh-DOPE did not integrate. 
581 Figure 7: Microscopy images showing the heterogeneous distribution of polar lipids and

582

583

584

585

586

587

588

589

590

591

592

593

594

595

596

597

598

599

600

601

602

603

604

605

606 glycosylated molecules (glycoproteins and glycolipids) in the buffalo milk fat globule membrane. In-situ observations performed by the combination of DIC optical microscopy and CLSM, using Rh-DOPE to label the phospholipids (red colour) and the lectin WGA-488 to stain the glycosylated molecules (green colour). (A) DIC image, (B) overlay of DIC and fluorescence with Rh-DOPE, (C) overlay of DIC image and fluorescence with WGA-488, (D) overlay of DIC image with Rh-DOPE and WGA-488, (E) equatorial cross section of the same fat globule showing the overlay of DIC image with Rh-DOPE and WGA-488. Images were captured using a 100x objective. The scale bars are indicated in the figures. White arrows correspond to non-fluorescent domains, green arrows correspond to glycosylated molecules located at the periphery or within a domain in which $\mathrm{Rh}-\mathrm{DOPE}$ did not integrate.

Figure 8: Microscopy images showing the heterogeneous distribution of polar lipids and glycosylated molecules (glycoproteins and glycolipids) in the buffalo milk fat globule membrane. In-situ observations performed by the combination of DIC optical microscopy and CLSM, using Rh-DOPE to label the phospholipids (red colour) and the lectin WGA-488 to stain the glycosylated molecules (green colour). (A) overlay of DIC image with Rh-DOPE and WGA-488, (B) overlay of DIC image with fluorescence with WGA-488, (C) overlay of DIC image and fluorescence with Rh-DOPE, (D) DIC image. Images were captured using a 100x objective. The scale bars are indicated in the figures. White arrows correspond to glycosylated molecules located at the periphery or within a domain in which Rh-DOPE did not integrate. 
607

608 FIGURES

609

610 Figure 1

611

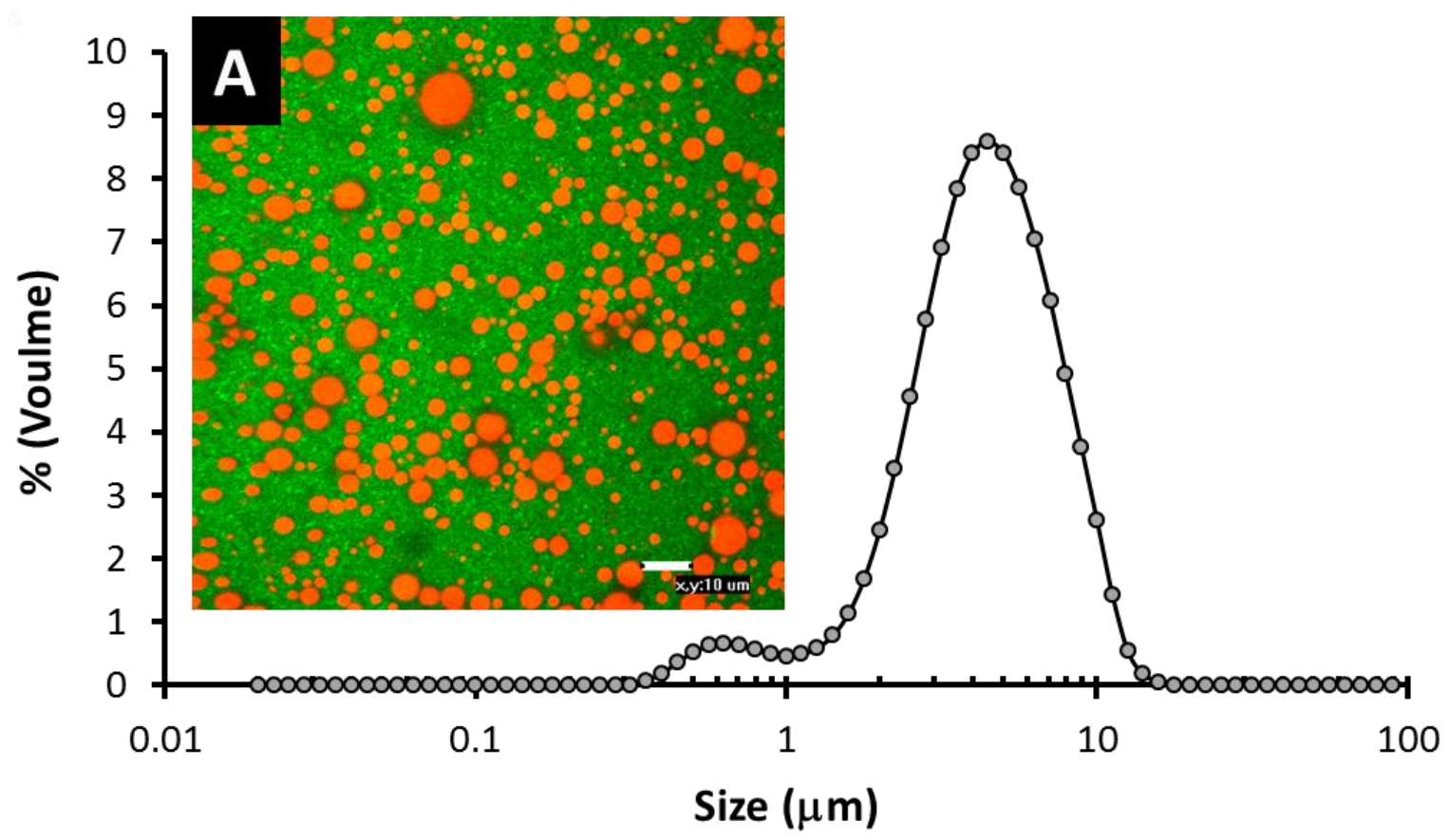

612

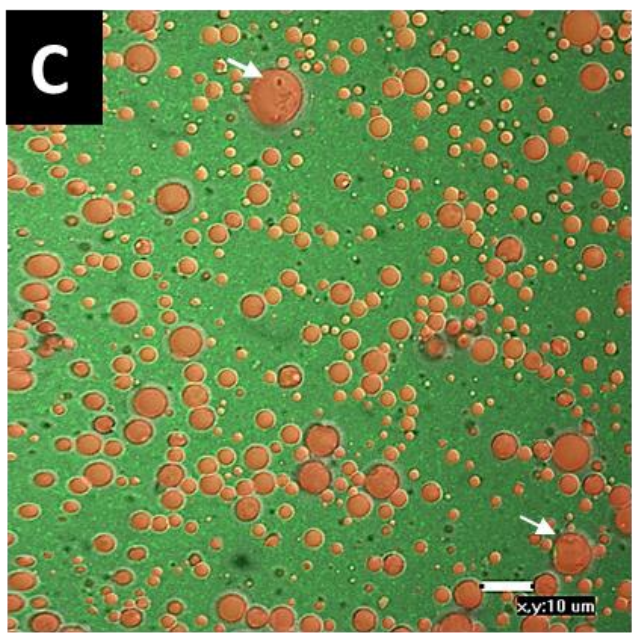

613 
$614 \quad$ Figure 2

615
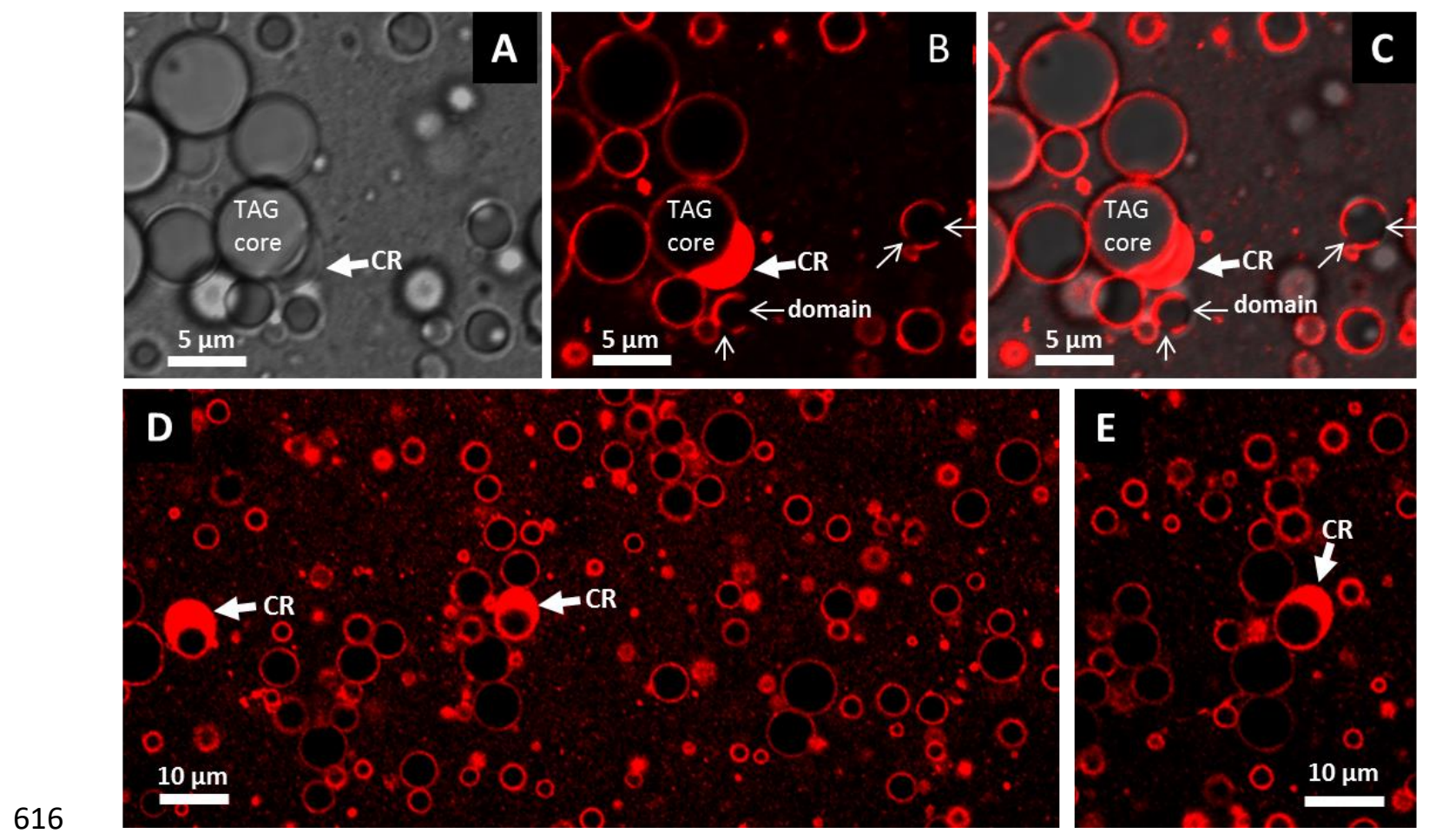

617

618 
$619 \quad$ Figure 3

620
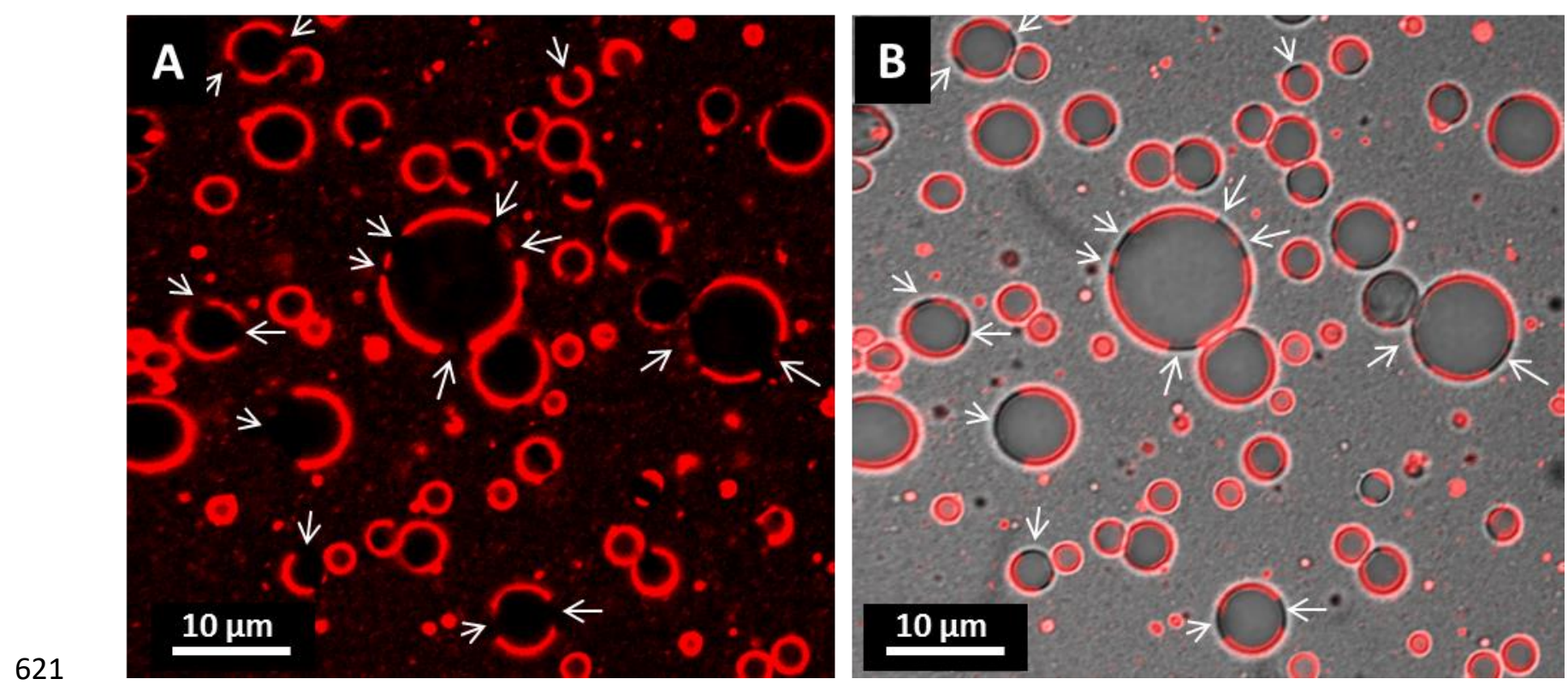

622

623 
$624 \quad$ Figure 4

625

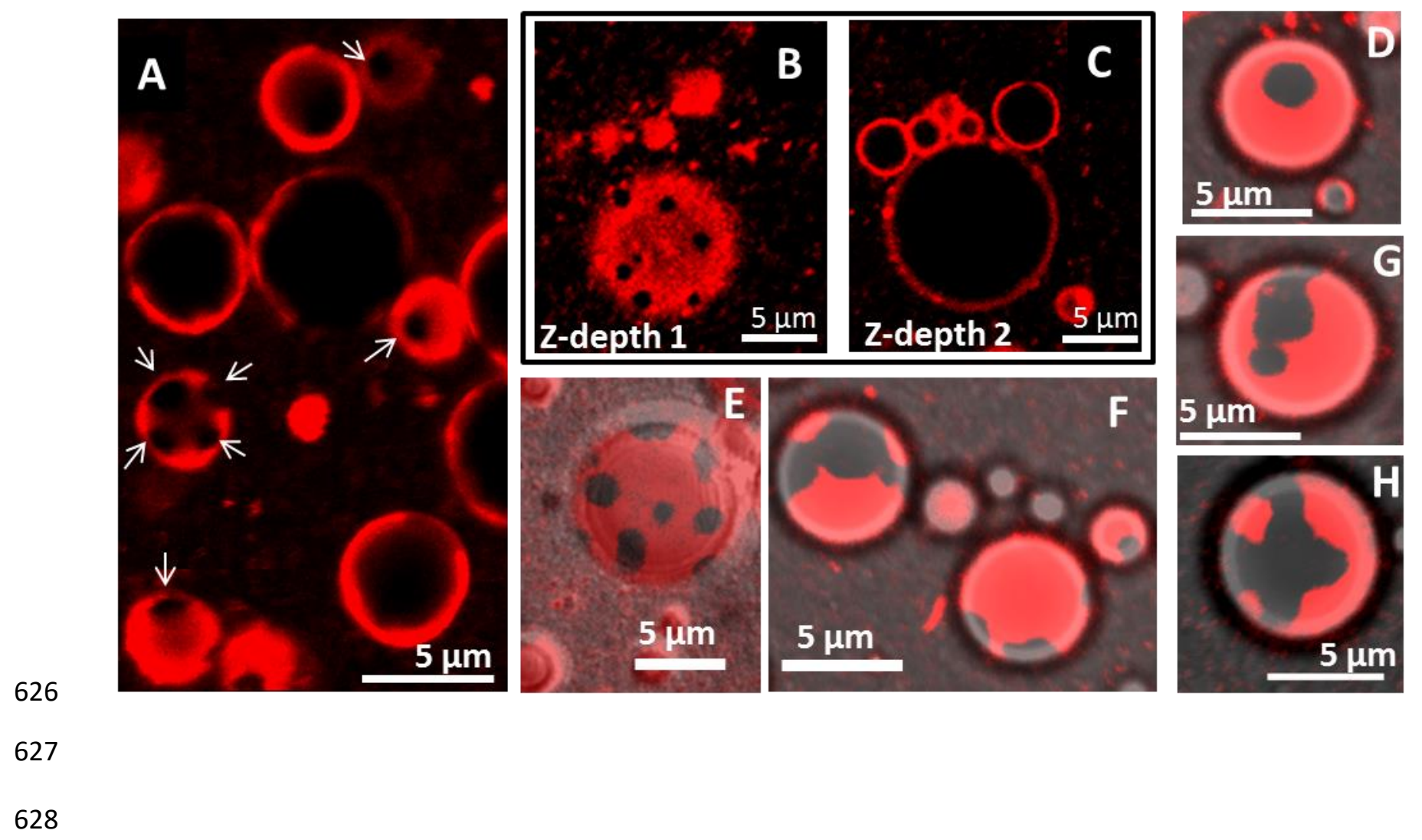


$629 \quad$ Figure 5

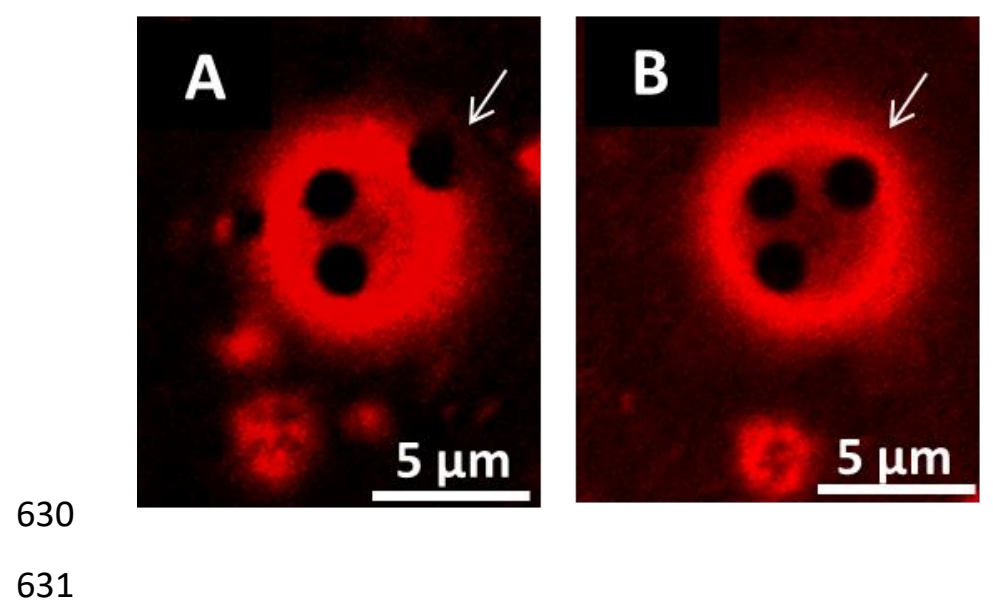

632

$633 \quad$ Figure 6

634

635

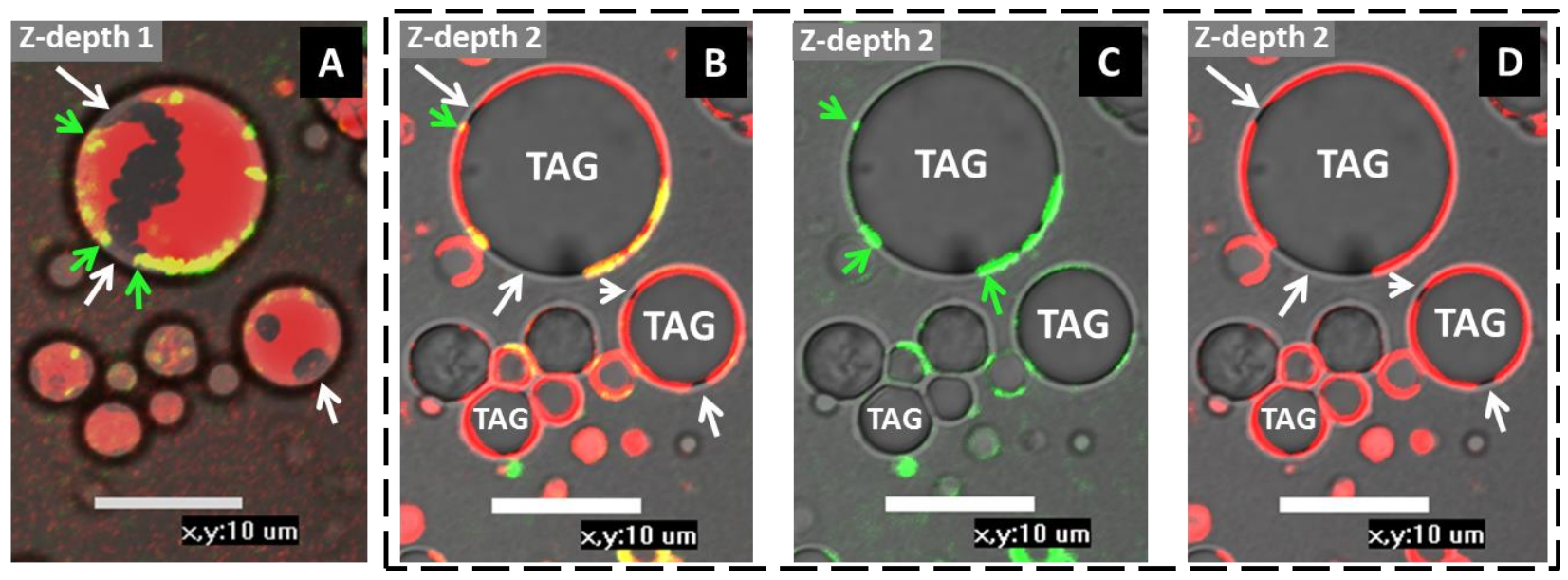

636

637

638

639

640

641

642

643 
$644 \quad$ Figure 7

645

646
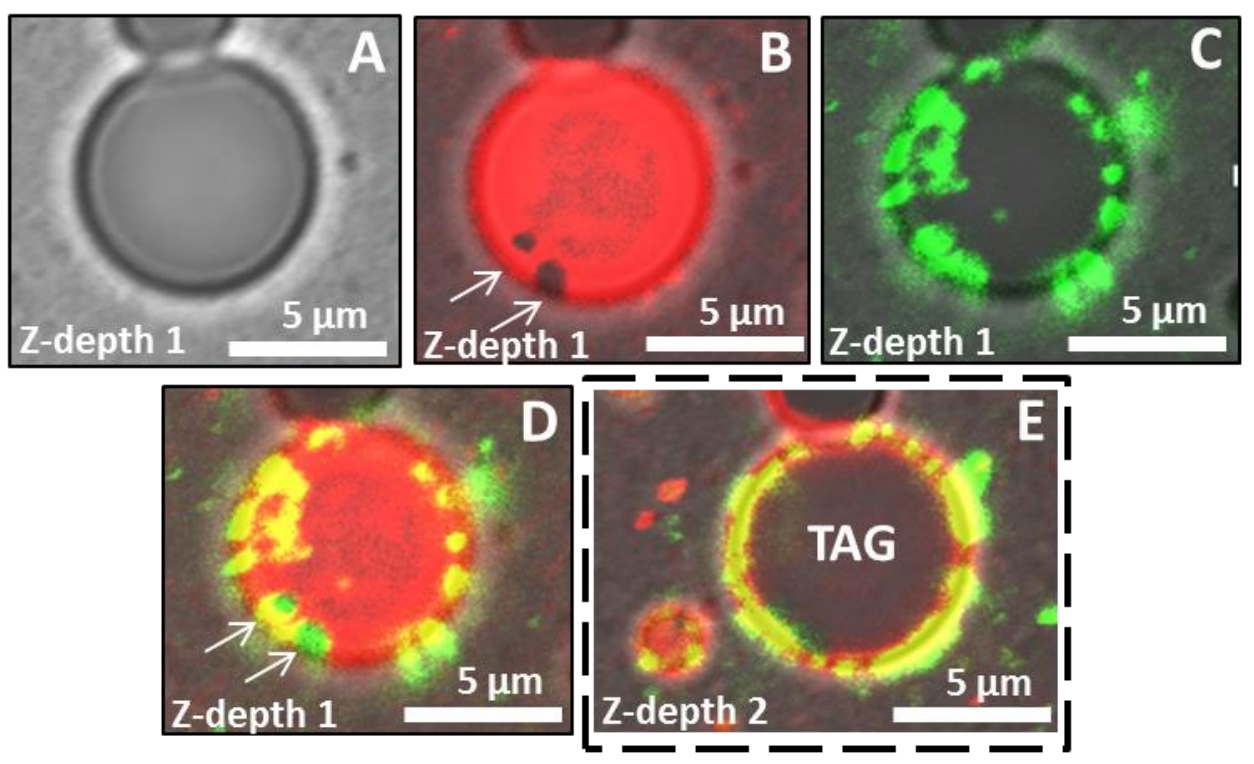

647

648 
$649 \quad$ Figure 8

650

651
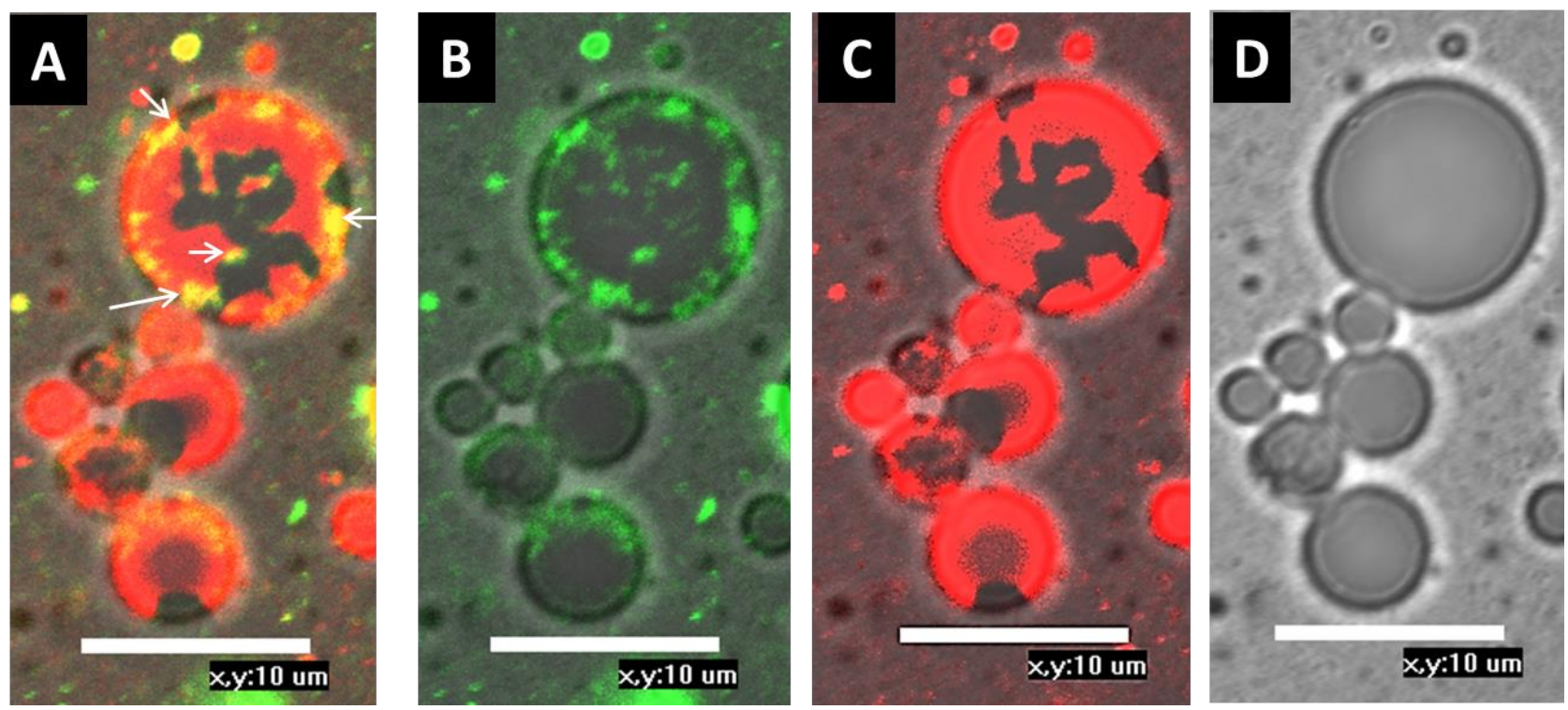